\title{
Effects of Subsequent Systemic Anticancer Medication Following First-Line Lenvatinib: A Post Hoc Responder Analysis from the Phase 3 REFLECT Study in Unresectable Hepatocellular Carcinoma
}

\author{
Angel Alsina ${ }^{a} \quad$ Masatoshi Kudo $^{b} \quad$ Arndt Vogel $^{c} \quad$ Ann-Lii Cheng ${ }^{d}$ Won Young Tak ${ }^{e}$ \\ Baek-Yeol Ryoo ${ }^{f}$ Thomas R. Jeffry Evans ${ }^{g}$ Carlos López López ${ }^{h} \quad$ Bruno Daniele $^{i}$ \\ Soamnauth Misirj ${ }^{j}$ Min Renk Namiki Izumil Shukui Qin ${ }^{m}$ Richard S. Finn ${ }^{n}$ \\ ${ }^{a}$ Transplant and Specialty Services, Tampa General Hospital, Tampa, FL, USA; ${ }^{b}$ Department \\ of Medicine, Kindai University, Osaka, Japan; ' Department of Gastroenterology, Hepatology, \\ and Endocrinology, Hannover Medical School, Hannover, Germany; ${ }^{\text {d Department of Medical }}$ \\ Oncology, National Taiwan University Hospital, and National Taiwan University Cancer Center,

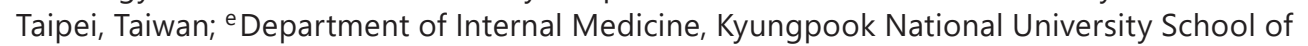 \\ Medicine, Daegu, Republic of Korea; ${ }^{f}$ Department of Oncology, Asan Medical Center, and \\ University of Ulsan College of Medicine, Seoul, Republic of Korea; 9 Institute of Cancer Sciences, \\ Beatson West of Scotland Cancer Centre, University of Glasgow, Glasgow, UK; ${ }^{\text {h }}$ Oncology \\ Service, Marqués de Valdecilla University Hospital, IDIVAL, Santander, Spain; 'Department of \\ Oncology, Azienda Ospedaliera G. Rummo, Benevento, Italy and Ospedale del Mare, Naples,

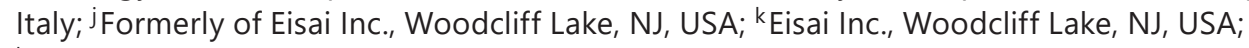 \\ 'Department of Gastroenterology and Hepatology, Musashino Red Cross Hospital, Musashino, \\ Tokyo, Japan; mPLA Cancer Center, Nanjing Bayi Hospital, Nanjing, Jiangsu, China; ${ }^{n}$ Geffen \\ School of Medicine, Department of Medicine, UCLA Medical Center, Santa Monica, CA, USA
}

\section{Keywords}

Lenvatinib · Sorafenib · Response status · Overall survival · Second-line treatment

\begin{abstract}
Introduction: Understanding the relationship between subsequent-line therapies and overall survival (OS) is important for maximizing OS for patients with hepatocellular carcinoma. $\mathbf{O b}$ jective: In this post hoc analysis, we investigated OS in lenvatinib- and sorafenib-treated patients from the REFLECT study, who then received subsequent anticancer medication during the survival follow-up period. Methods: The follow-up period commenced at the first offtreatment visit after stopping the study medication and continued until study termination,
\end{abstract}


withdrawal of consent, or death. OS and objective response rate were calculated for patients who did or did not receive poststudy anticancer medication for both treatment arms, as well as for the overall cohort. We investigated the subset of patients who responded to first-line treatment and subsequently received anticancer medication. Results: The OS for patients initially randomized to first-line lenvatinib (versus first-line sorafenib) and who then received any subsequent anticancer medication was 20.8 vs. 17.0 months (hazard ratio [HR] 0.87; 95\% Cl 0.67-1.14). The OS for patients who initially received first-line lenvatinib (versus first-line sorafenib) and who did not receive any subsequent anticancer medication was 11.5 vs. 9.1 months (HR 0.90; 95\% Cl 0.75-1.09). Responders to first-line lenvatinib who received subsequent medication had a median OS of 25.7 months ( $95 \% \mathrm{Cl} 18.5-34.6)$; responders to first line-sorafenib who received subsequent medication had a median OS of 22.3 months $(95 \% \mathrm{Cl}$ 14.6-not evaluable). Conclusions: In this post hoc analysis of all patients in the REFLECT study who received subsequent anticancer medication, OS was increased compared with patients who did not receive any subsequent anticancer medication. In a subset analysis of responders who had received subsequent anticancer medication, use of first-line lenvatinib led to a slightly longer median OS; more research is needed on the benefits of using first-line lenvatinib compared with sorafenib.

(C) 2019 The Author(s)

Published by S. Karger AG, Basel

\section{Introduction}

Hepatocellular carcinoma (HCC) is one of the most common cancers worldwide, with about 841,000 diagnoses and 782,000 deaths per year [1]. It is the most common form of liver cancer and is primarily associated with chronic infections by hepatitis $\mathrm{B}$ and $\mathrm{C}$ viruses. In 2015, 257 million people worldwide were reported as having the hepatitis B virus and 71 million people had the hepatitis $C$ virus [2]. These viruses often lead to cirrhosis, which limits treatment options for HCC $[3,4]$. Initially, sorafenib was the only first-line systemic therapy that improved overall survival (OS) for unresectable HCC (uHCC) [4, 5], until the recent (2018) approval of lenvatinib [6,7]. Lenvatinib is an oral multikinase inhibitor targeting both vascular endothelial growth factor receptors 1-3 and fibroblast growth factor receptors 1-4, in addition to platelet-derived growth factor receptor $\alpha$, RET, and KIT [8-11].

REFLECT was a randomized, open-label, phase 3 study that compared the efficacy and safety of lenvatinib versus sorafenib in first-line treatment of patients with uHCC [6]. Lenvatinib demonstrated a treatment effect on OS, statistically confirmed by noninferiority to sorafenib (the primary end point of median OS was 13.6 vs. 12.3 months; hazard ratio [HR] 0.92; 95\% CI 0.791.06) [6]. Lenvatinib resulted in statistically significant improvements in the secondary end points of progression-free survival (PFS), time to progression, and objective response rate (ORR) versus sorafenib (tested sequentially) [6]. Based on the outcomes from REFLECT, lenvatinib became the first agent approved in the first-line treatment setting for uHCC in over a decade [6].

The recent availability of new treatment options in first- and subsequent-line therapies has shifted the treatment paradigm for HCC, resulting in questions regarding appropriate medication choices and the timing and sequencing of anticancer therapies. There are relatively few studies directly examining the relationship between OS and subsequent-line therapies [12-17].

Understanding the relationship between subsequent-line therapies and OS is important for maximizing OS for patients with HCC. Therefore, in this post hoc analysis, we evaluated OS following first-line treatment with lenvatinib or sorafenib in patients with uHCC from REFLECT who received subsequent anticancer medication during the survival follow-up period. We also assessed the subset of patients who responded to lenvatinib or sorafenib during REFLECT, and subsequently received other systemic anticancer medication(s). 


\section{Liver Cancer}

\section{Methods}

Patients

Briefly, eligible patients were $\geq 18$ years of age and had histologically or cytologically uHCC with 1 or more measurable target lesions. Patients had Barcelona Clinic Liver Cancer stage B or C, Child-Pugh class A, and an Eastern Cooperative Oncology Group performance status (ECOG PS) score of 0 or 1 . Controlled blood pressure $(\leq 150 / 90 \mathrm{~mm} \mathrm{Hg}$ ) and adequate liver, bone marrow, blood, renal, and pancreatic functions were required. Exclusion criteria included $50 \%$ or higher liver occupation, clear bile duct invasion, invasion at the main portal vein (Vp4), or previous systemic therapy for HCC. Full patient eligibility criteria were previously published [6].

This study (NCT01761266) was conducted in accordance with the Declaration of Helsinki, all relevant Institutional Review Boards, and local laws. All patients provided written informed consent.

\section{Study Design and Treatment}

REFLECT was a phase 3, multicenter, randomized, open-label, noninferiority study comparing lenvatinib to sorafenib in patients with uHCC [6]. Patients were randomized 1:1 to receive either lenvatinib or sorafenib [6]. Treatment cycles were 28 days in length. Lenvatinib (Eisai Inc., Woodcliff Lake, NJ, USA) was administered orally once daily at a dosage of either $12 \mathrm{mg} /$ day (for baseline patient bodyweight $\geq 60 \mathrm{~kg}$ ) or $8 \mathrm{mg} /$ day (for baseline patient bodyweight $<60 \mathrm{~kg}$ ). In the case of lenvatinib-related adverse events, the dose could be reduced by increments of $4 \mathrm{mg}$ to a minimum dosage of $4 \mathrm{mg}$ every other day. Sorafenib (Bayer, Leverkusen, Germany) $400 \mathrm{mg}$ was administered orally twice daily. Modifications to sorafenib dosages were allowed based on regional prescribing information [6].

First-line treatment continued until disease progression, intolerable adverse events, or withdrawal of consent. The study follow-up period commenced at the first off-treatment visit (within 30 days of discontinuing the study drug for any reason) and continued until sponsor study termination, withdrawal of consent, or death [6]. All poststudy systemic anticancer therapies were reported until death or data cutoff (November 13, 2016) and were captured as part of the survival follow-up period.

\section{Study Objectives}

The primary end point was OS, as measured from randomization to patient death from any cause. Patients remaining alive at the end of the study were censored at data cutoff [6]. Secondary end points were PFS, time to progression, ORR, quality-of-life measurements, and plasma pharmacokinetics for lenvatinib [6]. These end points have previously been reported [6].

Post Hoc Subgroup Analyses

In this post hoc analysis, we investigated OS in lenvatinib- and sorafenib-treated patients from REFLECT who received subsequent anticancer medication during the survival follow-up period.

Patients who received nonsystemic subsequent anticancer treatments (e.g., transcatheter arterial chemoembolization) during the survival follow-up period were not excluded from the analysis. All patients who received subsequent systemic anticancer medication during the survival follow-up period were counted in the analysis as patients who received subsequent systemic medication, regardless of other treatments received. All other patients were counted in the analysis as patients who did not receive subsequent systemic anticancer medication. We also investigated the subset of patients who responded to lenvatinib or sorafenib during REFLECT, and subsequently received anticancer medication (includes patients who continued first-line medication and those who transitioned to a different medication) during the follow-up period. Responders were defined as patients who achieved either complete or partial tumor response as assessed by investigators in accordance with modified Response Evaluation Criteria In Solid Tumors (mRECIST), which do not require confirmation of response for randomized trials where ORR is not the primary end point [18]. Safety and tolerability data were not collected during the survival followup period.

\section{Statistical Methods}

Analyses were conducted for the subgroup of REFLECT patients who received subsequent anticancer medication. The HRs for OS were calculated using the Cochran-Mantel-Haenszel method, stratified by interactive response system stratification factors (region, macroscopic portal vein invasion, and ECOG PS) and are presented with 2-sided 95\% CI. Evaluations were performed using SAS version 9 or higher. 
Alsina et al.: Subsequent Anticancer Medication: A Post Hoc Analysis from REFLECT

Table 1. Poststudy medications received by patients during the survival follow-up period

\begin{tabular}{llc}
\hline $\begin{array}{l}\text { Medications taken during the } \\
\text { follow-up period, } n(\%)\end{array}$ & $\begin{array}{l}\text { First-line lenvatinib } \\
(n=478)\end{array}$ & $\begin{array}{l}\text { First-line sorafenib } \\
(n=476)\end{array}$ \\
\hline $\begin{array}{l}\text { Patients with any anticancer medication during } \\
\quad \text { survival follow-up period }\end{array}$ & $156(32.6)$ & $184(38.7)$ \\
Sorafenib & $121(25.3)$ & $56(11.8)$ \\
Fluorouracil & $20(4.2)$ & $26(5.5)$ \\
Cisplatin & $18(3.8)$ & $23(4.8)$ \\
Investigational drug & & $45(9.5)$ \\
Oxaliplatin & $15(3.1)$ & $22(4.6)$ \\
Doxorubicin & $14(2.9)$ & $19(4.0)$ \\
Capecitabine & $7(1.5)$ & $11(2.3)$ \\
Gemcitabine & $7(1.5)$ & $14(2.9)$ \\
Cabozantinib & $7(1.5)$ & $11(2.3)$
\end{tabular}

a 11 patients who received first-line lenvatinib and 15 patients who received first-line sorafenib went on to receive "investigational immunotherapies" as a subsequent medication.

\section{Results}

Subsequent Anticancer Medication(s) and Patient Status

Patient dispositions in REFLECT have been previously published [6]. A total of 340 patients were included in this post hoc subgroup analysis.

Of the 954 patients randomized to receive first-line lenvatinib $(n=478)$ or sorafenib $(n=476)$ in REFLECT, 340 received subsequent anticancer medication during the survival follow-up period: $156(32.6 \%)$ had received first-line lenvatinib and $184(38.7 \%)$ had received first-line sorafenib (Table 1). Of the patients who received subsequent anticancer medication, 60 who were randomized to first-line lenvatinib and 63 who were randomized to first-line sorafenib also received subsequent anticancer procedures (excluding radiotherapy). Of the patients who were treated with first-line lenvatinib, the most common subsequent anticancer medication was sorafenib (25.3\%; Table 1). Of the patients treated with first-line sorafenib, the most common subsequent anticancer medication beyond radiographic progression was also sorafenib (11.8\%; Table 1).

The biggest disparity between the first-line lenvatinib and first-line sorafenib groups was the number of patients enrolled in second-line clinical trials and randomized to subsequent investigational drugs or placebo: $3.1 \%$ of those who had received first-line lenvatinib versus $9.5 \%$ of those who had received first-line sorafenib (Table 1). The specific classes of these agents are unknown.

ECOG PS, Child-Pugh class, and liver function assessments for lenvatinib- and sorafenibtreated patients at the time of treatment discontinuation were similar between treatments (Table 2).

\section{OS for the REFLECT Population}

Patients in REFLECT randomized to first-line lenvatinib had a median OS of 13.6 months (95\% CI 12.1-14.9); patients randomized to first-line sorafenib had a median OS of 12.3 months (95\% CI 10.4-13.9 months; Table 3; Fig. 1, 2a) [6]. These data were previously published in Kudo et al. [6]. 
Table 2. Summary of patient status at treatment discontinuation

\begin{tabular}{|c|c|c|}
\hline Parameter & $\begin{array}{l}\text { Lenvatinib } \\
(n=451)\end{array}$ & $\begin{array}{l}\text { Sorafenib } \\
(n=451)\end{array}$ \\
\hline \multicolumn{3}{|l|}{ ECOG PS, $n(\%)$} \\
\hline 0 & $165(36.6)$ & 189 (41.9) \\
\hline 1 & $220(48.8)$ & $202(44.8)$ \\
\hline 2 & $42(9.3)$ & $39(8.6)$ \\
\hline$\geq 3$ & $22(4.9)$ & $20(4.4)$ \\
\hline \multicolumn{3}{|l|}{ Child-Pugh class, $n(\%)$} \\
\hline A & $339(75.2)$ & $345(76.5)$ \\
\hline $\mathrm{B}$ & $97(21.5)$ & $99(22.0)$ \\
\hline $\mathrm{C}$ & $13(2.9)$ & $6(1.3)$ \\
\hline ALT, nkat/L median (range) ${ }^{\mathrm{a}}$ & $533.33(50-12,100)$ & $650.0(100-18,417)$ \\
\hline AST, nkat/L median (range) ${ }^{\mathrm{a}}$ & $900.0(167-41,683)$ & $983.0(217-20,183)$ \\
\hline ALP, nkat/L median (range) ${ }^{a}$ & $2,233.3(417-30,950)$ & $2,333.3(467-24,283)$ \\
\hline Bilirubin, $\mu \mathrm{mol} / \mathrm{L}$ median (range) ${ }^{\mathrm{a}}$ & $12.8(2-643)$ & $12.7(3-408)$ \\
\hline Albumin, g/L median (range) ${ }^{\mathrm{a}}$ & $37.0(19-51)$ & $38.0(19-52)$ \\
\hline
\end{tabular}

${ }^{a}$ In the Western region, 2 patients from the lenvatinib arm and 1 patient from the sorafenib arm had no data. ALP, alkaline phosphatase; ALT, alanine aminotransferase; AST, aspartate aminotransferase; ECOG PS, Eastern Cooperative Oncology Group performance status.

Table 3. OS comparisons for patients receiving first-line lenvatinib or sorafenib for the overall REFLECT population, for patients who received subsequent anticancer medication, and for patients who responded to first-line medication and then received subsequent anticancer medication

\begin{tabular}{lclll}
\hline Parameter & $\begin{array}{l}\text { Number of } \\
\text { patients }\end{array}$ & $\begin{array}{l}\text { Median OS, } \\
\text { months (95\% CI) }\end{array}$ & $\begin{array}{l}\text { HR Lenvatinib } \\
\text { vs. Sorafenib }\end{array}$ & 95\% CI \\
\hline $\begin{array}{l}\text { Overall population } \\
\quad \text { Lenvatinib }\end{array}$ & 478 & $13.6(12.1-14.9)$ & 0.92 & $0.79-1.06$ \\
$\quad$ Sorafenib & 476 & $12.3(10.4-13.9)$ & & $0.67-1.14$ \\
$\begin{array}{l}\text { Received subsequent medication } \\
\quad \text { Lenvatinib }\end{array}$ & 156 & $20.8(15.1-23.5)$ & 0.87 & \\
$\quad$ Sorafenib & 184 & $17.0(14.4-19.1)$ & & NA \\
$\begin{array}{l}\text { Responded to first-line medication } \\
\text { and received subsequent anticancer medication }\end{array}$ & & & NA \\
$\quad$ Lenvatinib & 43 & $25.7(18.5-34.6)$ & NA & NA \\
$\quad$ Sorafenib & 16 & $22.3(14.6-\mathrm{NE})$ & $\mathrm{NA}$ & \\
\hline
\end{tabular}

HR, hazard ratio; NA, not applicable; NE, not estimable; OS, overall survival; REFLECT, a Multicenter, Randomized, Open-Label, Phase 3 Trial to Compare the Efficacy and Safety of Lenvatinib (E7080) Versus Sorafenib in First-Line Treatment of Subjects with Unresectable Hepatocellular Carcinoma.

OS for Patients Who Received Subsequent Anticancer Medication(s)

Approximately one-third of all patients from either treatment group received subsequent anticancer medication (Table 3; Fig. 1, 2b). Among patients who received subsequent anticancer medication, the median OS for patients randomized to first-line lenvatinib $(n=$ 156) versus first-line sorafenib $(n=184)$ was 20.8 vs. 17.0 months (HR 0.87 ; $95 \%$ CI 0.67 1.14; Table 3; Fig. 1, 2b). Among patients who did not receive subsequent anticancer medication $(n=614)$, the median OS for patients who received first-line lenvatinib versus first-line sorafenib was 11.5 vs. 9.1 months (HR 0.90; 95\% CI 0.75-1.09). 


\section{Liver Cancer}

Fig. 1. OS comparisons for patients receiving first-line lenvatinib or sorafenib for the overall REFLECT population and for patients who received subsequent anticancer medication during the survival follow-up period. OS, overall survival; REFLECT, A Multicenter, Randomized, Open-Label, Phase 3 Trial to Compare the Efficacy and Safety of Lenvatinib (E7080) Versus Sorafenib in First-Line Treatment of Subjects with Unresectable Hepatocellular Carcinoma; uHCC, unresectable hepatocellular carcinoma.

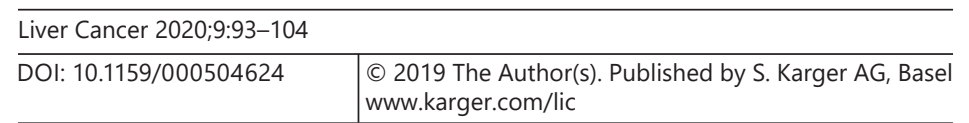

Alsina et al.: Subsequent Anticancer Medication: A Post Hoc Analysis from REFLECT

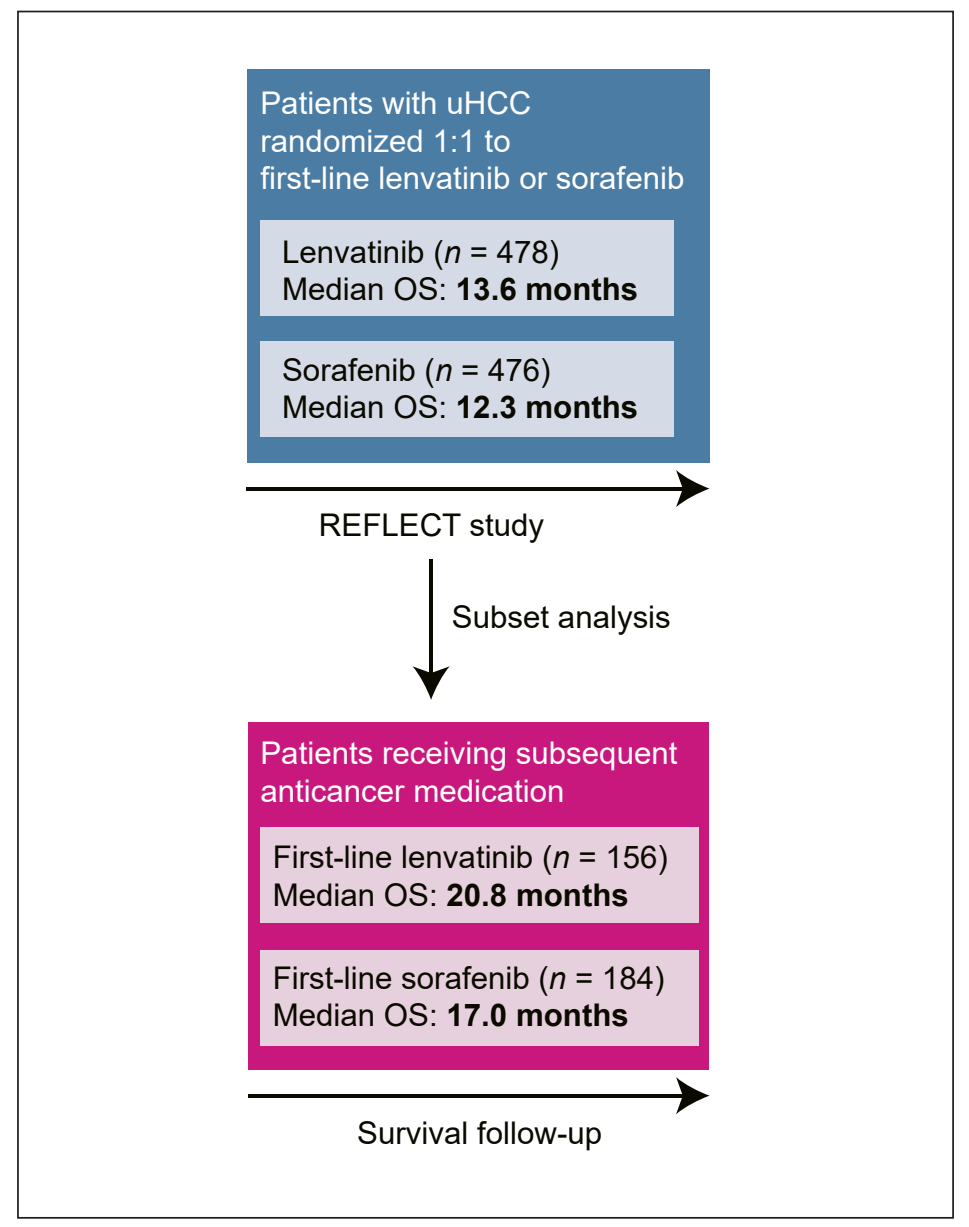

OS for First-Line Treatment Responders

First-line lenvatinib responders who received any subsequent anticancer medication $(n=43)$ had a median OS of 25.7 months (95\% CI 18.5-34.6; Table 3; Fig. 3a). First-line sorafenib responders who received any subsequent anticancer medication $(n=16)$ had a median OS of 22.3 months (95\% CI 14.6-not evaluable; Table 3; Fig. 3b). Lenvatinib responders who received subsequent sorafenib treatment $(n=35)$ had a median OS of 26.2 months $(95 \%$ CI 18.2-34.6; Fig. 4). Only 8 sorafenib responders received poststudy sorafenib treatment; therefore, survival estimates were not performed for this subgroup.

\section{Discussion}

The impact of the multiple recent approvals in the treatment of advanced HCC is challenging our assumptions on survival in the first-line setting. In REFLECT, lenvatinib had a positive effect on OS and was statistically confirmed to be noninferior to sorafenib, but did not show superiority despite its significant improvements in PFS, ORR, and time to progression [6]. Per investigator review using mRECIST, the ORR for lenvatinib was 24.1 vs. $9.2 \%$ for sorafenib [6]. A recent (2019) multivariate analysis by Kudo et al. [19] of REFLECT responders demonstrated a correlation between objective response and OS, similar to analyses from other large clinical trials in uHCC $[20,21]$, both by independent review using mRECIST. 


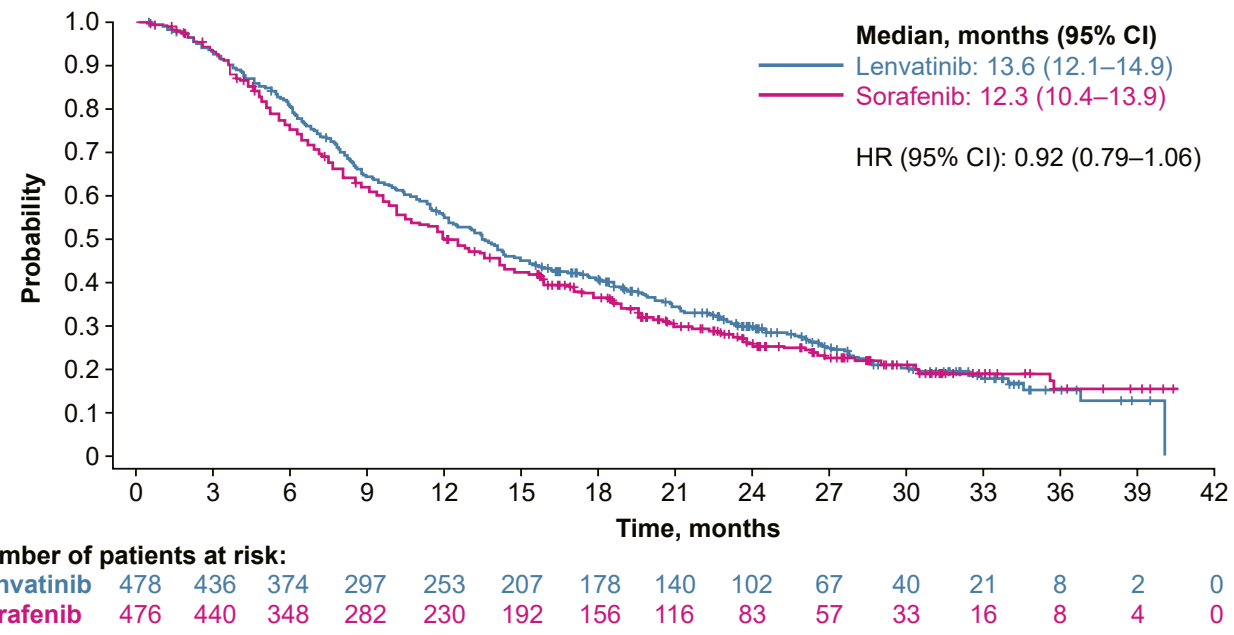

b

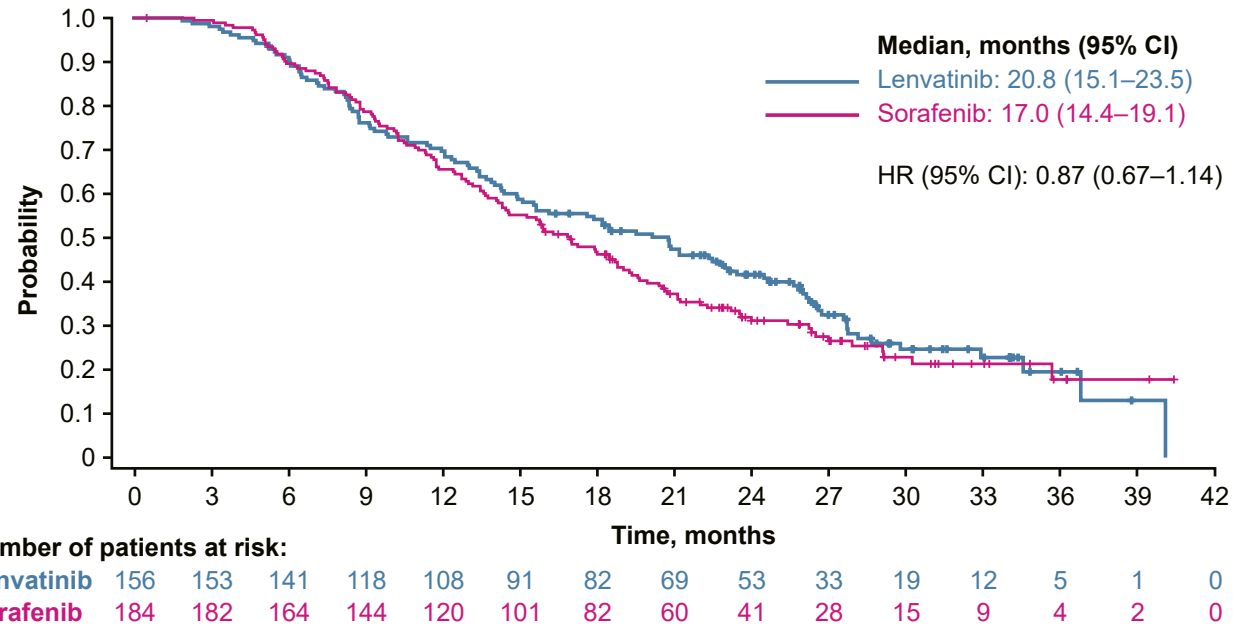

Fig. 2. Kaplan-Meier estimates of OS for (a) the overall REFLECT population by treatment arm (reprinted from Kudo et al. [6], Copyright 2018, with permission from Elsevier) and (b) patients from REFLECT who received subsequent anticancer medication by first-line treatment arm. HR, hazard ratio; REFLECT, A Multicenter, Randomized, Open-Label, Phase 3 Trial to Compare the Efficacy and Safety of Lenvatinib (E7080) Versus Sorafenib in First-Line Treatment of Subjects with Unresectable Hepatocellular Carcinoma; OS, overall survival.

This post hoc analysis, based on information captured in the survival follow-up period of REFLECT, adds additional support for investigating the importance of treatment sequence on OS. Approximately one-third of patients from both treatment arms in REFLECT received subsequent anticancer medication and at the time of treatment discontinuation, ECOG PS and liver function were similar for patients randomized to either treatment. Despite these similarities, in the subset of patients that received subsequent anticancer medication, patients randomized to first-line lenvatinib had numerically longer median OS than patients randomized to first-line sorafenib. While the number of first-line responders who received subsequent anticancer medication was too small to draw strong conclusions, those randomized to first-line lenvatinib also had longer 
a

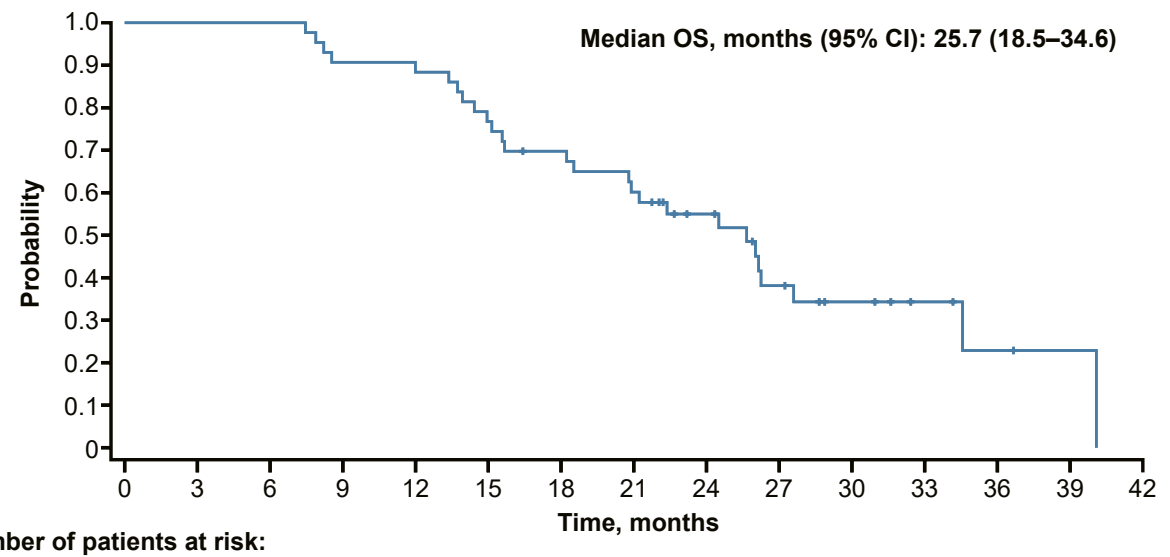

Number of patients at risk:

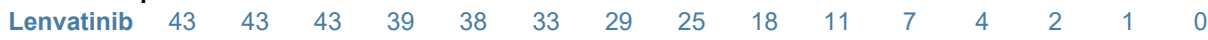

b

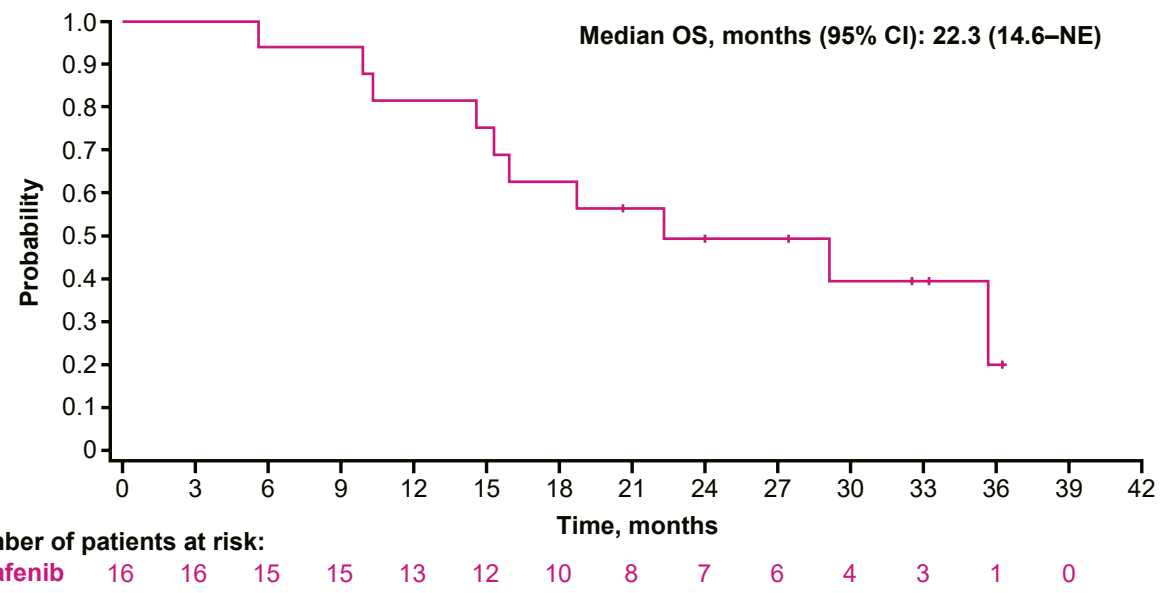

Fig. 3. Kaplan-Meier estimates of OS for (a) lenvatinib responders and (b) sorafenib responders who received any subsequent anticancer medication. NE, not estimable; OS, overall survival.

median OS compared with those randomized to first-line sorafenib. Interestingly, both treatment arms had similar median OS for the overall population. However, there were more patients who received subsequent anticancer medication following first-line sorafenib $(n=184)$ than patients who received first-line lenvatinib $(n=156)$, and this imbalance may be the cause of the similar OS between treatment arms in the overall population. Regardless of first-line treatment, patients who received subsequent anticancer medication had longer median OS as compared with patients who did not receive subsequent medication.

At the time of the REFLECT study (study period March 1, 2013 - November 13, 2016), currently common therapies such as regorafenib and nivolumab were in clinical trials requiring the failure of first-line sorafenib, which limited second-line investigational agent options for patients receiving first-line lenvatinib. Though approximately 3 -fold more patients treated with first line sorafenib went on to participate in clinical trials with investigational drugs, patients randomized to first-line lenvatinib who received subsequent medication had numerically longer median OS. The majority of patients who received first-line lenvatinib and then subsequent anticancer medication received second-line sorafenib, which was the only 


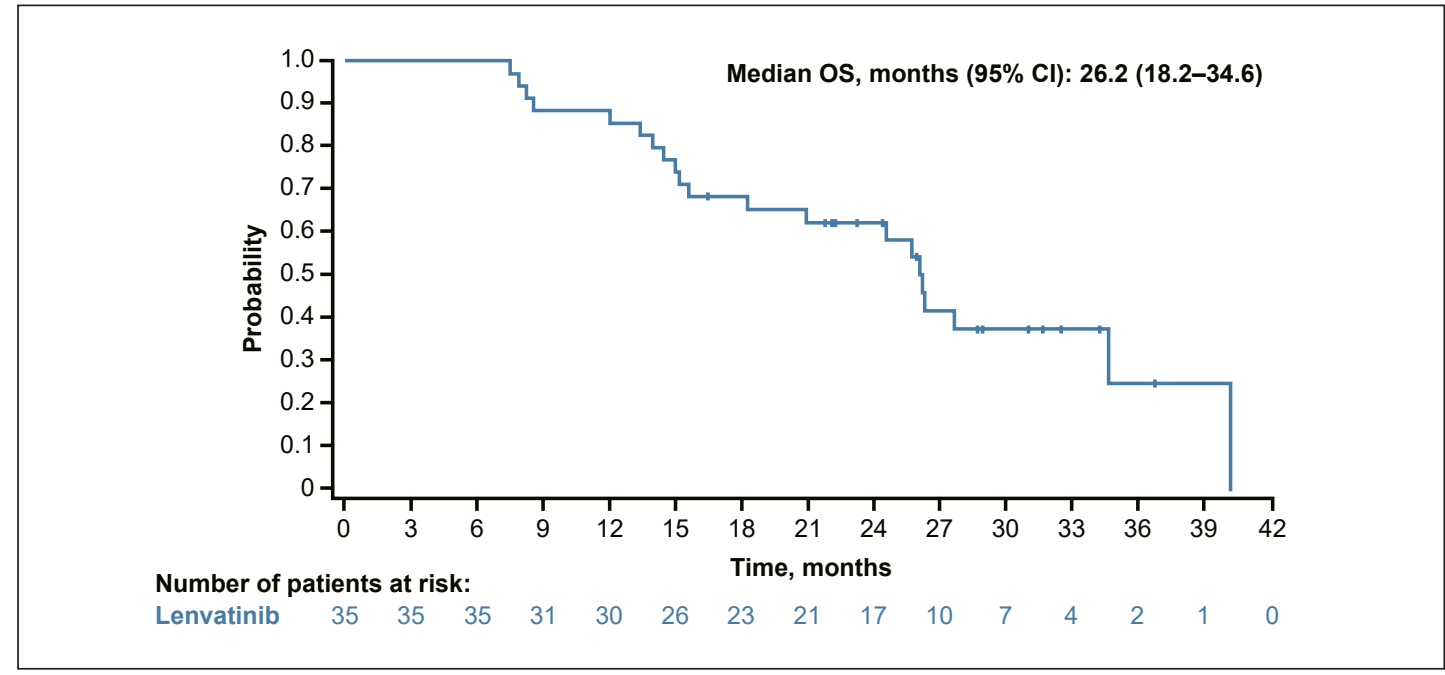

Fig. 4. Kaplan-Meier estimate of OS for lenvatinib responders who received subsequent sorafenib. OS, overall survival.

systemic treatment at the time with demonstrated activity in HCC [6]. Therefore, the difference in OS between patients who received first-line lenvatinib or sorafenib followed by subsequent anticancer medication may be attenuated simply by the requirement for the failure of first-line sorafenib to enter into investigational trials.

With the recent approval of many second-line treatment options (regorafenib, nivolumab, pembrolizumab, cabozantinib, and ramucirumab) for uHCC [22, 23], it is important to assess treatment patterns for patient benefit. There are many hurdles to getting a patient with uHCC to second-line treatment. Disease characteristics, such as ECOG status and poor liver function, can have a significant outcome on trial results, as they can decrease tolerance of the study medication and increase the incidence of AEs [24].

The study of regorafenib after sorafenib in patients with HCC (RESORCE) was a phase 3 trial showing that patients with HCC who progressed on sorafenib and were then randomized to regorafenib had a median OS of 10.6 months (95\% CI 9.1-12.1) compared with 7.8 months (6.3-8.8) for those then randomized to a placebo (NCT01774344 [25]). However, patients who participated in RESORCE could not have any systemic treatments for HCC prior to sorafenib, and they must have tolerated sorafenib and only discontinued due to disease progression [25]. Additionally, they had to have a Child-Pugh Class A, an ECOG PS of 0 or 1, and a life expectancy of at least 3 months [25]. These criteria may have improved the chance of patients tolerating regorafenib, which then resulted in improved OS [24].

The patient population of REFLECT was much more varied, yet the median survival for patients randomized to first-line lenvatinib followed by subsequent medication, especially for responders, is the highest reported to date in a phase III trial for uHCC. These data suggest that first-line lenvatinib followed by subsequent systemic anticancer medication could be an important treatment sequence that may lead to longer OS. This sequence may have even greater survival benefits for responders to first-line lenvatinib, though the proportion of responders who received subsequent anticancer medication was small compared with the overall REFLECT population. We also recognize that our post hoc analyses are limited patients were not prerandomized to the subsequent medications they received, and there could be selection bias confounding the results. Given the exploratory nature of these analyses, the results presented here are descriptive and should be interpreted with caution. 


\section{Liver Cancer}

Liver Cancer 2020;9:93-104

DOI: $10.1159 / 000504624$

(c) 2019 The Author(s). Published by S. Karger AG, Base www.karger.com/lic

Alsina et al.: Subsequent Anticancer Medication: A Post Hoc Analysis from REFLECT

\section{Acknowledgments}

Medical writing assistance was provided by Caroline Leitschuh, PhD, of Oxford PharmaGenesis Inc., Newtown, PA, USA, with funding provided by Eisai Inc., Woodcliff Lake, NJ, USA, and Merck Sharp and Dohme Corp., a subsidiary of Merck and Co. Inc., Kenilworth, NJ, USA.

\section{Statement of Ethics}

All procedures performed in the source studies involving human participants were in accordance with the ethical standards the International Conference on Harmonisation of Pharmaceuticals for Human Use, the United States Code of Federal Regulations, the European Good Clinical Practice Directive, the European Clinical Trial Directive, the Japanese Pharmaceutical Affairs Law, with the 1964 Helsinki Declaration and its later amendments or comparable ethical standards, and all relevant Institutional Review Boards, and local laws. All patients of these source studies had provided written informed consent.

\section{Data Sharing Statement}

The data will not be available for sharing at this time as the data are commercially confidential. However, Eisai will consider written requests to share the data on a case-by-case basis.

\section{Disclosure Statement}

A.A.: reports honoraria, consulting or advisory fees, and speakers' bureau fees from Eisai. M.K.: reports honoraria and consulting or advisory fees from Bayer AG and Eisai Co., Ltd.; honoraria from Bayer AG, Bristol-Myers Squibb, EA Pharma Co., Ltd., Eisai Co., Ltd., and Merck Sharp \& Dohme; research funding from Bayer AG, Daiichi Sankyo Co., Ltd., Chugai Pharmaceutical Co., Ltd., Merck Sharp \& Dohme, Otsuka Pharmaceutical Co., Ltd., Sumitomo Dainippon Pharma Co., Ltd., and Taiho Pharmaceutical and is the Editor-in-Chief of Liver Cancer. A.V.: reports consulting or advisory fees from Novartis International, Delcath Systems, Eli Lilly and Company, Roche Holding AG, Amgen Inc., Bayer AG, and Baxalta; honoraria from Novartis International, Roche Holding AG, Bayer AG, Sanofi S.A., Amgen Inc., Delcath Systems, Eli Lilly and Company, Bristol-Myers Squibb, and Merck Sharp \& Dohme; personal fees from Bayer AG, Roche Holding AG, and Ipsen Corporate; and research funding from Novartis International. A.-L.C.: reports consulting or advisory fees from Bristol-Myers Squibb, Ono Pharmaceutical Co., Ltd., Novartis International AG, Bayer AG, Merck Group, and Merck Sharp \& Dohme; and is an associate editor of Liver Cancer. W.Y.T.: reports consulting or advisory fees and speakers' bureau fees from Bayer HealthCare Pharmaceuticals, Korea-Gilead Sciences, Inc., AbbVie Korea, Samil Pharmaceutical Co., Yuhan Co., Ltd.; consulting or advisory fees from Bukwang Pharmaceutical Co., Ltd., Eisai Korea Inc., and Ono Pharma Korea Co., Ltd.; and speakers' bureau fees from Merck Sharp \& Dohme Korea. B.-Y.R. and S.Q.: declare no conflicts of interest. T.R.J.E.: reports honoraria from Eisai Co., Ltd. for advisory boards and speaker's bureau; support from Eisai Co., Ltd. for sponsored clinical trials; honoraria for advisory boards and speakers' bureau fees from Bristol-Myers Squibb, Bayer AG, GlaxoSmithKline, and Roche/Genentech; honoraria for advisory boards from Celgene Corporation, Karus Therapeutics, Baxalta, TC BioPharm, and Immunova Therapeutics; support for sponsored clinical trials from Bristol-Myers Squibb, GlaxoSmithKIine, Roche/ Genentech, Celgene Corporation, TC BioPharm, Merck Sharp \& Dohme, Novartis, e-Therapeutics, Vertex, Verastem, Daiichi, AstraZeneca, Basilea, Immunocore, and Chugai; support to attend international scientific conferences from Bristol-Myers Squibb, Roche/Genentech, Bayer AG, Merck Sharp \& Dohme, and Eisai Co., Ltd. C.L.L.: reports consulting or advisory fees and research funding from Bristol-Myers Squibb, Merck Sharp \& Dohme, Eisai Co., Ltd., Merck Group, Servier, Sanofi S.A., Roche/Genentech, EXELISIS, Daiichi-Sankyo, Ipsen, and AstraZeneca; and consulting or advisory fees from Bayer AG, Amgen Inc., Novartis, and Pfizer Inc. B.D.: reports honoraria from Bristol-Myers Squibb and Bayer AG; consulting or advisory fees from Bayer AG, Eisai Co., Ltd., Eli Lilly and Company, Ipsen Corporate, and Merck Sharp \& Dohme; and personal fees from Amgen Inc., Bayer AG, Bristol-Myers Squibb, Celgene Corporation, and Sanofi S.A. S.M.: employed by Eisai Inc., while this study was conducted and when the manuscript was first 
drafted. M.R.: employed by Eisai Inc. N.I.: reports being an associate editor of Liver Cancer. R.S.F.: reports consulting or advisory fees and research funding from Bayer AG, Bristol-Myers Squibb, Eisai Co., Ltd., Eli Lilly and Company, Merck Group, Novartis International AG, and Pfizer Inc.; consulting or advisory fees from AstraZeneca plc; and is on the editorial board Liver Cancer.

\section{Funding Sources}

This study and editorial support services related to the manuscript were funded by Eisai Inc., Woodcliff Lake, NJ, and Merck Sharp \& Dohme Corp., a subsidiary of Merck \& Co., Inc., Kenilworth, NJ, USA.

\section{Previous Presentations}

Gastrointestinal Cancers Symposium of the American Society of Clinical Oncology; January 17-19, 2019; San Francisco, CA, USA.

HCC-UK Annual Conference; March 14-15, 2019; Birmingham, UK.

Asia-Pacific Primary Liver Cancer Expert Congress; August 29-31, 2019; Hokkaido, Japan.

\section{Author Contribution}

S.M. and M.R.: made substantial contributions to the design of the study and the acquisition, analysis, and interpretation of data for the work. All authors made substantial contributions to revising the manuscript critically for important intellectual content, and made final approval of the version to be published. All authors agree to be accountable for all aspects of the work in ensuring that questions related to the accuracy or integrity of any part of the work are appropriately investigated and resolved.

\section{References}

1 Bray F, Ferlay J, Soerjomataram I, Siegel RL, Torre LA, Jemal A. Global cancer statistics 2018: GLOBOCAN estimates of incidence and mortality worldwide for 36 cancers in 185 countries. CA Cancer J Clin. 2018 Nov;68(6): 394-424.

2 World Health Organization. Global hepatitis report, 2017 [cited 9 Sep 2019]. Available from: https://www. who.int/hepatitis/publications/global-hepatitis-report2017/en/.

3 El-Serag HB. Epidemiology of viral hepatitis and hepatocellular carcinoma. Gastroenterology. 2012 May; 142(6):1264-1273.e1.

4 El-Serag HB. Hepatocellular carcinoma. N Engl J Med. 2011 Sep;365(12):1118-27.

5 Llovet JM, Ricci S, Mazzaferro V, Hilgard P, Gane E, Blanc JF, et al.; SHARP Investigators Study Group. Sorafenib in advanced hepatocellular carcinoma. N Engl J Med. 2008 Jul;359(4):378-90.

6 Kudo M, Finn RS, Qin S, Han KH, Ikeda K, Piscaglia F, et al. Lenvatinib versus sorafenib in first-line treatment of patients with unresectable hepatocellular carcinoma: a randomised phase 3 non-inferiority trial. Lancet. 2018 Mar;391(10126):1163-73.

7 Lenvima (lenvatinib) [prescribing information]. Woodcliff Lake: Eisai Inc.; 2018.

8 Tohyama O, Matsui J, Kodama K, Hata-Sugi N, Kimura T, Okamoto K, et al. Antitumor activity of lenvatinib (e7080): an angiogenesis inhibitor that targets multiple receptor tyrosine kinases in preclinical human thyroid cancer models. J Thyroid Res. 2014;2014:638747.

9 Yamamoto Y, Matsui J, Matsushima T, Obaishi H, Miyazaki K, Nakamura K, et al. Lenvatinib, an angiogenesis inhibitor targeting VEGFR/FGFR, shows broad antitumor activity in human tumor xenograft models associated with microvessel density and pericyte coverage. Vasc Cell. 2014 Sep;6(1):18.

10 Matsui J, Yamamoto Y, Funahashi Y, Tsuruoka A, Watanabe T, Wakabayashi T, et al. E7080, a novel inhibitor that targets multiple kinases, has potent antitumor activities against stem cell factor producing human small cell lung cancer H146, based on angiogenesis inhibition. Int J Cancer. 2008 Feb;122(3): 664-71.

11 Matsui J, Funahashi Y, Uenaka T, Watanabe T, Tsuruoka A, Asada M. Multi-kinase inhibitor E7080 suppresses lymph node and lung metastases of human mammary breast tumor MDA-MB-231 via inhibition of vascular endothelial growth factor-receptor (VEGF-R) 2 and VEGF-R3 kinase. Clin Cancer Res. 2008 Sep;14(17):545965 . 
12 Pfisterer J, Weber B, Reuss A, Kimmig R, du Bois A, Wagner U, et al.; AGO-OVAR; GINECO. Randomized phase III trial of topotecan following carboplatin and paclitaxel in first-line treatment of advanced ovarian cancer: a gynecologic cancer intergroup trial of the AGO-OVAR and GINECO. J Natl Cancer Inst. 2006 Aug;98(15):103645.

13 Hayashi H, Okamoto I, Taguri M, Morita S, Nakagawa K. Postprogression survival in patients with advanced non-small-cell lung cancer who receive second-line or third-line chemotherapy. Clin Lung Cancer. 2013 May; 14(3):261-6.

14 Turkmen E, Erdogan B, Kodaz H, Hacibekiroglu I, Onal Y, Uzunoglu S, et al. Post progression survival analysis of metastatic gastric and gastroesophageal junction cancer patients after second-line treatment. Acta Gastroenterol Belg. 2016 Apr-Jun;79(2):211-5.

15 Kotake M, Miura Y, Imai H, Mori K, Sakurai R, Kaira K, et al. Post-progression survival associated with overall survival in patients with advanced non-small-cell lung cancer receiving docetaxel monotherapy as second-line chemotherapy. Chemotherapy. 2017;62(4):205-13.

16 Zhu AX, Finn RS, Edeline J, Cattan S, Ogasawara S, Palmer D, et al.; KEYNOTE-224 investigators. Pembrolizumab in patients with advanced hepatocellular carcinoma previously treated with sorafenib (KEYNOTE224): a non-randomised, open-label phase 2 trial. Lancet Oncol. 2018 Jul;19(7):940-52.

17 Finn RS, Ryoo BY, Merle P, Kudo M, Bouattour M, Lim HY, et al. Results of KEYNOTE-240: phase 3 study of pembrolizumab (Pembro) vs best supportive care (BSC) for second line therapy in advanced hepatocellular carcinoma (HCC). J Clin Oncol. 2019;37(15 Suppl):4004.

18 Lencioni R, Llovet JM. Modified RECIST (mRECIST) assessment for hepatocellular carcinoma. Semin Liver Dis. 2010 Feb;30(1):52-60.

19 Kudo M. Objective response by mRECIST is an independent prognostic factor of overall survival in systemic therapy for hepatocellular carcinoma. Liver Cancer. 2019 Mar;8(2):73-7.

20 Lencioni R, Montal R, Torres F, Park JW, Decaens T, Raoul JL, et al. Objective response by mRECIST as a predictor and potential surrogate end-point of overall survival in advanced HCC. J Hepatol. 2017 Jun;66(6): 1166-72.

21 Meyer T, Palmer DH, Cheng AL, Hocke J, Loembé AB, Yen CJ. mRECIST to predict survival in advanced hepatocellular carcinoma: analysis of two randomised phase II trials comparing nintedanib vs sorafenib. Liver Int. 2017 Jul;37(7):1047-55.

22 Bteich F, Di Bisceglie AM. Current and future systemic therapies for hepatocellular carcinoma. Gastroenterol Hepatol (N Y). 2019 May;15(5):266-72.

23 Mahipal A, Tella SH, Kommalapati A, Lim A, Kim R. Immunotherapy in hepatocellular carcinoma: is there a light at the end of the tunnel? Cancers (Basel). 2019 Jul;11(8):E1078.

24 Sun W, Cabrera R. Systemic treatment of patients with advanced, unresectable hepatocellular Ccarcinoma: emergence of therapies. J Gastrointest Cancer. 2018 Jun;49(2):107-15.

25 Bruix J, Qin S, Merle P, Granito A, Huang YH, Bodoky G, et al.; RESORCE Investigators. Regorafenib for patients with hepatocellular carcinoma who progressed on sorafenib treatment (RESORCE): a randomised, doubleblind, placebo-controlled, phase 3 trial. Lancet. 2017 Jan;389(10064):56-66. 